\title{
La Gestión Urbana Ambiental En América Latina, 1970 - 2017
}

\section{David J. Edelman}

Departamento de Planificación Facultad de Diseño, la Arquitectura, el Arte y la Planificación Universidad de Cincinnati Cincinnati, OH, EE.UU.

\section{Micaela Schuster}

Instituto para Estudios sobre la Vivienda y Planeamiento Urbano (IHS),

Erasmus Universidad, Rotterdam, NL

\section{Janett Said}

Departamento de Planificación Facultad de Diseño, la Arquitectura, el Arte y la Planificación Universidad de Cincinnati Cincinnati, OH, EE.UU.

Doi: 10.19044/esj.2017.v13n30p63 URL:http://dx.doi.org/10.19044/esj.2017.v13n30p63

\begin{abstract}
While Urban Environmental Management has received increasing worldwide attention in the last 50 years, much of the international attention to this growing field has focused, as is the case with most scientific fields, on the English language literature. Nevertheless, much professional work of interest has been taking place in Latin America, where the problems of the urban environment have been identified early and considered major difficulties in the development of this heavily urbanised region. Consequently, the purpose of the research that this article summarises is to address the Spanish and Portuguese language literature written in Latin America on Latin America itself to identify the trends in the field that have emerged, and are continuing to emerge, there and, eventually, to determine what lessons they offer to other regions.
\end{abstract}

Keywords: Urban Environmental Management, Latin America, urban planning

\section{Resumen}

Mientras que la gestión ambiental urbana ha recibido creciente atención mundial en los últimos 50 años, gran parte de la atención internacional hacia este campo creciente se ha centrado, como es el caso con la mayoría de los campos de la ciencia, en la literatura de lengua inglesa. No obstante, gran parte del trabajo profesional de interés ha tenido lugar en América Latina, donde los problemas del medio ambiente urbano han sido 
identificados tempranamente y examinó las principales dificultades en el desarrollo de esta región muy urbanizados. Por lo tanto, el propósito de la investigación que este artículo resume es abordar la literatura de lengua española y portuguesa en América Latina escrita en América Latina para identificar las tendencias en el campo que han emergido y continúan surgiendo $\mathrm{y}$, eventualmente, para determinar qué lecciones ofrecen a otras regiones.

Palabras-claves: Gestión Ambiental Urbana, América Latina, ciudades, planificación urbana, sostenibilidad, español, portugués

\section{Introducción}

El tema de la gestión urbana ambiental ha recibido considerable atención desde 1970 en los últimos años, tanto en los países desarrollados, en donde emergió como sujeto de investigación académica e interés profesional, y en los países subdesarrollados, en donde también forma parte de la agenda de donantes bilaterales y multilaterales. Como campo de estudio, la gestión urbana ambiental, se asemeja más al planeamiento o ingeniería, que a la geografía, economía o sociología; y representa una visión integradora de los problemas ambientales en la ciudad, y cada vez más, a escala regional.

Los problemas ambientales involucran a todos sectores (manufactura, servicios, hogar, etc.), a todos los sistemas (de transporte, de agua, de higiene, etc.), a todos los niveles (local, regional, central) y a todos los actores (gobierno, ONG, organizaciones comunales y el sector privado). Las soluciones a estos problemas son de extrema complejidad ya que los responsables en coordinar el planeamiento, implementación y la gestión de los procesos deben estar capacitados para comunicarse con especialistas de varias disciplinas y profesiones (como, por ejemplo, biología, química, ingeniería, planeación, administración pública, ciencias sociales y derecho).

En cuanto a los aspectos teoréticos y conceptuales de esta materia, se puede decir que hasta la fecha no ha habido demasiados aportes. El término gestión urbana ambiental, fue mencionado por primera vez por Brian J. L. Berry y Frank E. Horton como título de su libro sobre planeamiento y control de la polución. Otros autores que han contribuido al tema incluyen a Carl R. Bartone (1989) y Josef Leitmann (1994) del Banco Mundial; los geógrafos canadienses William Rees (1996), Richard Stren (1992), Rodney White y Joe Whitney (1992), Kenneth J. Button y David W. Pearce (1989), el planificador Richard Meier y sus asociados en Berkley (1981), Jorge E. Hardoy y David Satterthwaite del IIED en el RU (1989), y el escritor inglés y realizador de películas Herbert Giradet (1992), S. Galilea (1994), E. Ocon (¡994), E. Leff (1998), R.G.J. Boon et al. (2001), Miriam Alfie Cohen 
(2007), L.M. Santana Rodríguez et al. (2010), M.C. Sánchez et al., y P. Avila Garcia (2012), entre otros.

Sin embargo, muy poco se ha contribuido para desarrollar una teoría comprensiva que abarque todo el proceso de las interacciones urbanas ambientales. Tal vez incluso más sorprendente es el hecho de que solo hay un texto universidad en la materia (Leitmann, 1999), y está fuera de la impresión. Pareciera ser que existen buenos argumentos para que la comunidad académica lleve a cabo una interesante investigación. Una necesidad prioritaria enfatizado en la literatura durante los últimos 50 años ha sido el desarrollo de un nuevo marco para políticas, por medio del cual exista la posibilidad de cambio. Y es por ello que la creación de una teoría comprensiva es tan importante.

Ante la falta de tal teoría se podría, sin embargo, considerar a los problemas actuales como la teoría que facilitaría un marco para el desarrollo de políticas. En Latinoamérica para la década de 1990, los problemas urbanos ambientales más importantes podrían ser, en principio, agrupados, en principio, en tres categorías, (1) los problemas que comprenden la agenda marrón, (2) los que comprenden la agenda gris y (3) las dificultades que implica la gestión de ambas. Estas categorías difieren de aquellas cuestiones ambientales que involucran a la totalidad del mundo animal y vegetal, incluyendo a los seres humanos (la agenda verde), tales como la preservación de las especies, el calentamiento de la tierra, conservación de los bosques, etc. En Latinoamérica, estos temas de agenda verde proporcionaron el tema inicial desde el que se introdujo la sostenibilidad, y un enfoque, por muy inadecuado que sea, para orientar temas más complejos como los de las agendas marrones y grises (Vásconez, 2000).

La agenda marrón, en cambio, se centra exclusivamente en el ser humano $\mathrm{y}$ en los efectos que los sistemas humanos han creado principalmente (aunque no exclusivamente) sobre el hombre. Las cuestiones preocupantes de esta agenda incluyen la provisión de agua; saneamiento y sistemas de alcantarillado; basura; emisiones de los autos, camiones, autobuses y de gases domésticos de baja magnitud; congestionamientos y hacinamientos humanos; ruido y la ocupación de tierras que presentan características sensibles a las actividades humanas. Si bien estos son problemas comunes a todas las ciudades, hay otros que son característicos sólo de algunas ciudades en particular. Podemos mencionar, en éste sentido, a la Ciudad de México y de Santiago que presentan altos grados de contaminación del aire generada por los vehículos a motor; mientras que Lima tiene grandes dificultades en proveer agua potable a las nuevas corrientes de inmigración urbana y rural.

También podemos hacer una distinción entre la agenda marrón y los problemas comprendidos en la agenda gris, que tienen que ver con la 
polución industrial, del aire, del suelo y del agua, incluyendo las emisiones de las fábricas y la basura peligrosa.

$\underline{\text { Por muchos }} \underline{\text { años }}$ en el contexto de la gestión urbana ambiental in América Latina, nos preguntamos si deberíamos tratar en forma separada los problemas de la agenda marrón, originados en su mayoría por la pobreza, de los de la agenda gris, relacionados directamente con los sistemas de producción. Los dos grupos de problemas ambientales deben ser resueltos en forma diferente, ya que algunas ciudades se caracterizan por uno solo mientras que otras ciudades tienen ambos (Edelman, 1998).

Un tercer problema crítico es el que tiene que ver con la gestión en sí. En este sentido Mitlin y Satterwaite (1996) han afirmado que el medio ambiente urbano no puede ser gestionado exclusivamente dentro de los confines políticos de una ciudad, sino que se requieren marcos locales, regionales y nacionales para una gestión efectiva. En el contexto de América Latina, el ámbito local no se refiere solamente a los municipios sino más que nada a las aglomeraciones urbanas compuestas de varios municipios. A modo de ejemplo podemos mencionar el caso de Lima, la cual está dividida en 40 municipios diferentes, siendo cada uno de ellos responsable de la recolección de basura. En zonas calificadas como clase alta, este sistema funciona bien, pero en zonas de clase baja no. Otro caso es el de Bolivia, que, bajo el nuevo sistema de restitución de responsabilidades administrativas, cada uno de los 311 municipios tiene a su cargo también sus alrededores. Sin embargo, muchos de ellos no cuentan con instituciones suficientes para manejar la diversidad de los problemas ambientales que enfrentan a diario. En otros países, la gestión ambiental se mantiene centralizada y tampoco se les otorga demasiada atención a las distintas instituciones involucradas en los procesos de gestión ambiental; las cuales a su vez cuentan con su propio mandato y responsabilidades geográficamente determinadas.

La literatura más reciente de la gestión ambiental urbana sugiere un cambio de la discusión inicial compartimentada y temática sobre los desafíos para las ciudades, tales como las dificultades de tratar estos temas por separado. En la actualidad, más trabajo reconoce la complejidad y la interconexión de estas cuestiones y, en consecuencia, el debate se centra en la necesidad de que el sector público haya llevado a un cambio sistemático para abordar estos problemas urbanos apremiantes de manera preventiva y no reactiva.

Nos preguntamos entonces, ¿Qué tipo de estructura institucional flexible y pragmática es la necesaria o apropiada para manejar los problemas urbanos ambientales en América Latina? En este respecto, sería apropiado analizar los niveles conceptuales de trabajo desarrollados por Mitlin y Satterthwaite, así como los distintos modelos organizativos en cada uno de 
dichos niveles; ya que lo que puede implementarse en un país, no necesariamente podrá implementarse en otro.

Más allá de estos problemas, de carácter técnico y de gestión, podemos mencionar un cuarto problema que se refiere a la falta de conocimiento y prevención de los problemas urbanos ambientales, tanto por parte del público en general como de los políticos. Mientras que la mayoría del público se ve afectado por su ignorancia sobre la pobreza, los funcionarios políticos no lo están. Para ellos, la realidad de sus circunstancias políticas simplemente hace que sus prioridades sean alocadas hacia otras preocupaciones, que por lo general caen en lo económico. En este contexto, el punto importante de discusión sería el de cómo elevar el nivel de atención hacia cuestiones urbanas ambientales, tanto por el público como por sus representantes.

Es un hecho que el interés internacional por esta materia nueva y de rápida evolución se ha centrado en la literatura de lengua inglesa, así como ha ocurrido con cualquier otra materia científica. Es decir, la mayor parte de los conocimientos sobre cuestiones medioambientales emergentes se producen en centros de investigación y desarrollo tecnológico del "Norte", señalando una falta de capacidad en la autodeterminación científica y tecnológica para alimentar el desarrollo de políticas sostenibles en los países latinoamericanos (Fernández, 2000). Mientras que, paradójicamente, la mayor parte del trabajo profesional ha tenido lugar en América Latina, en donde los problemas del medio ambiente urbano han sido identificados hace tiempo y donde el desarrollo de esta región, altamente urbanizada, presenta grandes dificultades. El expresidente del Banco Interamericano de desarrollo (IADB), Enrique Iglesias, ha manifestado entre otros temas relevantes al sector urbano en América Latina, la importancia de las ciudades, democratización, descentralización y la infraestructura. Todos estos temas convergen en lo que denominamos el ambiente urbano.

El propósito de investigación del presente artículo es el de analizar la literatura de lenguas española y portuguesa escrita en América Latina y sobre América Latina misma, para identificar las tendencias emergentes en el campo y eventualmente determinar las experiencias que ofrece la región para otras regiones.

\section{Metodología}

El trabajo descripto ha sido el resultado de un estudio basado sobre Internet y se divide en dos fases. Fase I cubre el periodo desde 1970, antes de Hábitat I en Vancouver (1976), hasta 1998, dos años después Hábitat II en Estambul (1996). Este trabajo ha sido publicado en Español (Schuster y Edelman, 2002) e inglés (Schuster y Edelman, 2003). La fase II se basa, modifica y amplía el trabajo anterior para cubrir el período desde 1998 hasta 
2017. Habitat III en Quito (2016) trajo la discusión global de la urbanización y la gestión ambiental urbana al corazón de América Latina. Muchos de los que han intentado, sabrán que navegar por Internet para conseguir cuanta información se pueda, y en países en los cuales este tipo de fuente de información no siempre está al alcance de la mano, no es algo fácil de alcanzar. Sin embargo, se ha podido lograr un trabajo fructuoso. El primer paso en este inicial estudio (Fase I), consistió en la identificación de la mayor cantidad de referencias bibliográficas posibles y encontrar sumarios o anotaciones de las mismas. De toda la información recibida fue posible, en una etapa inicial, dividir las tendencias literarias sobre el tema de gestión urbana ambiental en América Latina en cuatro periodos históricos:
a) $1970 / 1979$
b) $1980 / 1989$
c) $1990 / 1992$
d) $1993 / 1998$

Sin embargo, el análisis de fase II identificó un período histórico adicional con sus propias características únicas; es decir:

e) $1999 / 2017$

Para corroborar las conclusiones extraídas del análisis de las referencias bibliográficas encontradas vía Internet, se volvió a buscar en Internet una vez más para ambas fases, pero esta vez revistas especializadas en el tema. Se tomaron en cuenta los mismos períodos históricos, en orden de corroborar la existencia de un paralelismo entre las tendencias demostradas por los libros y aquellas demostradas por las revistas. Sin embargo, el enfoque aplicado no fue el mismo. Durante la investigación bibliográfica fue posible comprobar que la mayoría de los libros de interés se encuentran en las páginas de Internet de bibliotecas nacionales, las cuales a su vez nos brindan acceso a otras redes del país en cuestión. En estos casos, Internet provee suficientes fuentes de información, y ya no es necesario buscar en otras páginas. Un ejemplo, es el caso de la Biblioteca del Congreso de los Estados Unidos. Por el contrario, no ocurre lo mismo con las revistas o información periódica. Estas fuentes de información son nacionales, internacionales o regionales, y pueden variar desde publicaciones comerciales para industrias, publicaciones periódicas de ciertas organizaciones o revistas publicadas por instituciones profesionales de carácter académico. Por otra parte, la literatura periódica cambia con el tiempo con nuevas revistas y revistas emergentes y las más antiguas desapareciendo. Esto es especialmente cierto en las publicaciones digitales basadas en Internet. Así, una serie de sitios de Internet consultados en la fase i ya no existían en la fase II, pero eran, sin embargo, sustituidos por sitios similares. Muchos cambios han tenido lugar con Internet en el período de 47 años de este estudio 
Para poder copar con esta cantidad variada de material y para mantenerse a la par de las evoluciones en el campo del medio ambiente, una búsqueda país-por-país era y sigue siendo esencial. Sin embargo, la región latinoamericana es muy amplia y no todos los países o ciudades están involucradas activamente en la investigación o práctica profesional en la gestión urbana ambiental. Teniendo en cuenta este aspecto, se realizó una selección de siete países hispanohablantes. Éstos varían en tamaño, ubicación y grado de desarrollo, pero incluyen la mayoría de la población urbana de la región; además de haber reconocido la severidad de los problemas del medio ambiente urbano y de contar con profesionales e instituciones académicas dedicados al manejo de los mismos. Los países seleccionados son: Argentina, Brasil, Chile, Colombia, Cuba, México y Perú. Alguna literatura adicional se dirige periféricamente a el Salvador, Ecuador, Venezuela y Latinoamérica como región.

\section{EI Contexto Latinoamericano}

Las tendencias sobre la gestión urbana ambiental no pueden ser analizadas sin antes comprender el contexto histórico en general de la región. Este es el caso, por lo menos, de América Latina en donde la evolución de la literatura de interés para esta investigación, es un claro reflejo de la historia de la región. Tal como Michael Redclift y David Goodman (1991) han citado:

Aún antes de su oficial descubrimiento, América Latina y Central eran vistas, para los europeos, como la puerta hacia la India; y pasaron a representar una fuente inacabable de todo lo que los europeos necesitaban. Dentro de sus extensas junglas y cadenas montañosas se encontraba un caudal de recursos naturales que podía ser transformado en riqueza. Sólo se requirió de la ingenuidad y de una gran cantidad de manos laboriosas, para convertir esos tesoros en beneficios en forma material tangible. La imagen de América Latina en el arte europeo, así como lo demuestran Baddeley y Fraser (1989), está absorta con la diversidad del continente. Esta distancia, usualmente visualizada como la distancia entre los valores primitivos y los civilizados, justificaba las conquistas de los españoles y portugueses a América, y les daba el derecho de poner el sello final de la conquista a través de la destrucción de aquellas vidas que vivían más cerca de la naturaleza, la población indígena del continente.

Los vestigios de las imágenes históricas evocadas en el párrafo anterior proporcionan el contexto que aún hoy día nos ayuda a entender muchas cuestiones sobre desarrollo de esta región. Incluyendo aquellas cuestiones relacionadas con el medio ambiente en general y el medio 
ambiente urbano en particular. Cuestiones de índole social, político, cultural $\mathrm{y}$ ambiental han sido fuertemente delineadas por los procesos económicos internos y externos que tuvieron lugar en América Latina; y son estos procesos económicos los que contribuyeron de gran manera al tipo de desarrollo de la región.

A diferencia de lo ocurrido en Francia (finales de 1960), no es posible identificar un momento histórico específico en el cual se haya fundado un movimiento ecológico organizado, aunque la Conferencia de las Naciones Unidas sobre el medio ambiente, celebrada en río en 1992, tuvo un impacto sustancial en el discurso de sostenibilidad de la gestión ambiental urbana en la región. Por el contrario, los temas ambientales han ido surgiendo gradualmente debido a las presiones extranjeras y como consecuencia de la dependencia económica con Europa, pero por sobre todo de los Estados Unidos. Las primeras obras literarias publicadas sobre temas ambientales se referían a temas tecnológicos y no sobre la sostenibilidad de la tecnología - tendencia que ya está cambiando (ej. en los 90’s). Un ejemplo es el caso de la agricultura. Cualquier estudio realizado sobre la de América Latina desde 1942, no puede dejar de reconocer la importancia del uso de la tierra para la agricultura y el arrendamiento (en forma de mano de obra de la población indígena). Esta práctica y forma de cultivo industrial extractivo y no sustentable, reemplazó los cultivos de una gran variedad de plantas naturales de los indígenas, como el tabaco, mandioca, maíz y papa, así como también el uso equilibrado y balanceado de los recursos para la producción; sistema implementado por la población indígena.

La historia nos supo demostrar que una evolución similar ocurriría durante el período de la industrialización. Durante las primeras décadas de este siglo, muchos países de América Latina lograron importantes pasos en su industrialización, un proceso que se encontraba en grado avanzado en países como Argentina, Brasil, Chile y México hacia 1940. El desarrollo industrial de las economías más grandes de América Latina estaba basado en la sustitución de importaciones, un modelo de desarrollo facilitado por Europa Occidental y los Estados Unidos. Los gobiernos de la región asumieron que el crecimiento industrial permitiría a la región reducir su dependencia con el exterior y eventualmente, desarrollar un gran mercado interno para los productos de sus propias industrias (Redclift y Goodman, óp. cit.).

Este modelo, como bien es conocido por la mayoría, fracasó. Pero, por sobre todo creó elevados patrones de consumismo, además de incrementar las expectativas del consumidor. La región continuó desarrollándose, por supuesto, pero dentro de un modelo de dependencia que continúa hoy día, junto con la globalización de la economía mundial. 
Para América Latina, la globalización significa que la participación en la economía mundial y sus mercados internacionales depende, entre otros requerimientos, de la observancia de los estándares medio ambientales. En los esfuerzos por participar del mercado internacional, los países de la región comenzaron a desarrollar perfiles de políticas medio ambientales, para no ser discriminados del resto del mundo. El reconocimiento de las limitaciones de sus propias instituciones y necesidades de gestión, así como el reconocimiento del potencial en recursos naturales, se convirtió en una de las tareas prioritarias para los gobiernos de América Latina. En la práctica, esto significó, darle una mayor prioridad a la gestión ambiental de los recursos naturales; definir en forma clara las responsabilidades institucionales y asegurar la representación de los distintos intereses; participar en la elaboración del concepto de estándares internacionales de gestión medio ambiental (por ej. ISO 14000) y explorar el uso de los instrumentos económicos para el manejo de los problemas ambientales. Nada de esto cae dentro de lo que denominamos 'urbano' y las tendencias urbanas serán analizadas en forma detallada más adelante. No debemos olvidar, que cada país tiene su propia historia, estructura social e instituciones, y por lo tanto el modelo adoptado variará según cada país. Algunos países lograrán más que otros en este respecto. Así y todo, se puede deducir de la investigación realizada en la literatura sobre la gestión urbana ambiental, que la tendencia regional hacia una mejor gestión ambiental es evidente y clara.

\section{Tendencias en la literatura}

Las tendencias de la literatura sobre gestión urbana ambiental reflejan la evolución de la historia y la sensibilidad ambiental descriptas en la sección anterior. Podemos visualizar las tendencias y sus evoluciones con la ayuda de la Figura 1. 
Figura 1: Tendencias de la literatura latinoamericana sobre gestión urbana ambiental

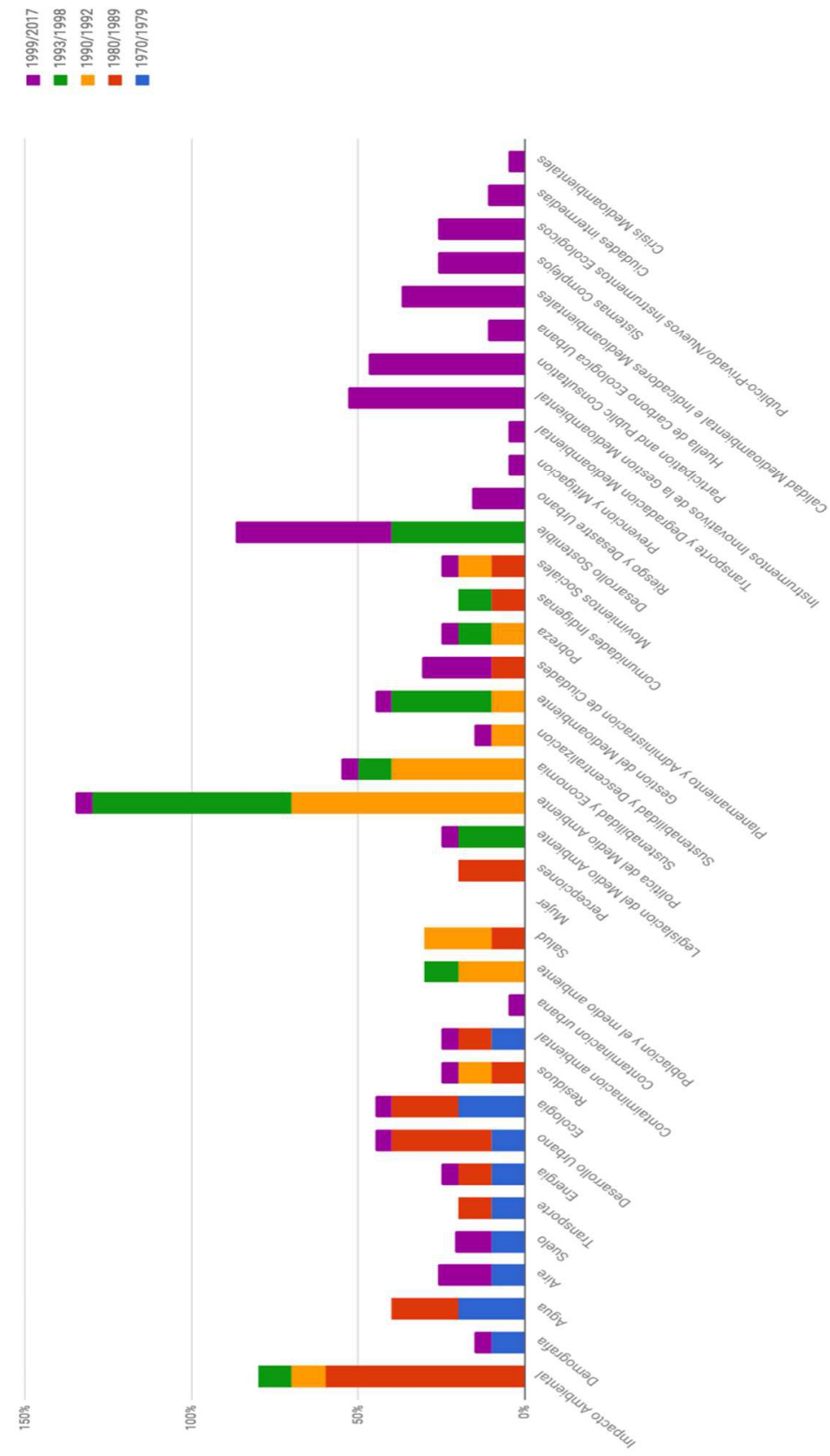

Cada uno de los períodos históricos mencionados en la Sección 2 y sus correspondientes temas ambientales, característicos de la literatura de dichos períodos, pueden visualizarse en la Figura 1 y son analizados a continuación. Las palabras en el eje $X$ de la figura, representan una lista clave de los temas más importantes que surgieron durante la búsqueda en 
Internet, y representan así mismo la importancia de dichos temas en un período histórico dado. Se contó cada palabra clave, y su incidencia en la literatura latinoamericana en un período histórico dado. Así mismo, los libros citados también fueron revisados y seleccionados como representativos de las mismas.

Utilizando el método de búsqueda proporcionado por Internet, nos permite acceder a una lista inicial de palabras claves que nos llevan a la referencia bibliográfica. El método de búsqueda, sin embargo, también nos muestra resultados secundarios, de poca relevancia a la investigación. Por ello se revisaron los contenidos de las referencias y se analizó su correlación con la palabra clave introducida en la búsqueda. Las palabras claves que resultarían en referencias totalmente irrelevantes fueron descartadas, y es así como se llegó a la lista de la Figura 1. Una vez que las referencias bibliográficas fueron revisadas, se realizó el mismo procedimiento para las revistas y publicaciones periódicas. Todas las referencias que aparecieron en esta investigación son el resultado de dicho proceso; y forman en conjunto la imagen de los temas más importantes de cada período, así como las tendencias que fueron evolucionando a lo largo de la historia en América Latina. La lista de palabras claves junto con el porcentaje de incidencia para cada uno de los cinco períodos, que se muestra en la figura 2 del Anexo de la Sección 11 al final de este informe.

Figura 2: Frecuencia de ocurrencia de palabras clave por periodo*

\begin{tabular}{|c|c|c|c|c|c|}
\hline & $\mathbf{1 9 7 0 / 1 9 7 9}$ & $\mathbf{1 9 8 0} / \mathbf{1 9 8 9}$ & $\mathbf{1 9 9 0 / 1 9 9 2}$ & $\mathbf{1 9 9 3 / 1 9 9 8}$ & $\begin{array}{c}\mathbf{1 9 9 9} \\
\mathbf{2 0 1 7}\end{array}$ \\
\hline Impactos Ambientales & $0 \%$ & $\mathbf{6 0} \%$ & $10 \%$ & $10 \%$ & $\mathbf{0 \%}$ \\
\hline Demografía & $\mathbf{1 0} \%$ & $0 \%$ & $0 \%$ & $0 \%$ & $\mathbf{5 \%}$ \\
\hline Agua & $\mathbf{2 0} \%$ & $\mathbf{2 0} \%$ & $0 \%$ & $0 \%$ & $\mathbf{0 \%}$ \\
\hline Aire & $10 \%$ & $0 \%$ & $0 \%$ & $0 \%$ & $\mathbf{1 6 \%}$ \\
\hline Tierra y Suelo & $10 \%$ & $0 \%$ & $0 \%$ & $0 \%$ & $\mathbf{1 1 \%}$ \\
\hline Transporte & $10 \%$ & $10 \%$ & $0 \%$ & $0 \%$ & $\mathbf{0 \%}$ \\
\hline Energía & $10 \%$ & $10 \%$ & $0 \%$ & $0 \%$ & $\mathbf{5 \%}$ \\
\hline Desarrollo Urbano & $10 \%$ & $\mathbf{3 0} \%$ & $0 \%$ & $0 \%$ & $\mathbf{5 \%}$ \\
\hline Ecología & $\mathbf{2 0} \%$ & $\mathbf{2 0 \%}$ & $0 \%$ & $0 \%$ & $\mathbf{5 \%}$ \\
\hline Residuos & $0 \%$ & $10 \%$ & $10 \%$ & $0 \%$ & $\mathbf{5 \%}$ \\
\hline Polución Ambiental & $10 \%$ & $10 \%$ & $0 \%$ & $0 \%$ & $\mathbf{5 \%}$ \\
\hline Contaminación Urbana & $0 \%$ & $0 \%$ & $0 \%$ & $0 \%$ & $\mathbf{5 \%}$ \\
\hline Población y Medio & $0 \%$ & $0 \%$ & $\mathbf{2 0 \%}$ & $10 \%$ & $\mathbf{0 \%}$ \\
\hline Ambiente. & $0 \%$ & $10 \%$ & $\mathbf{2 0 \%}$ & $0 \%$ & $\mathbf{0 \%}$ \\
\hline Salud & $0 \%$ & $0 \%$ & $0 \%$ & $0 \%$ & $\mathbf{0 \%}$ \\
\hline Mujer & $0 \%$ & $\mathbf{2 0 \%}$ & $0 \%$ & $0 \%$ & $\mathbf{0 \%}$ \\
\hline Percepciones & & & & \\
\hline
\end{tabular}




\begin{tabular}{|c|c|c|c|c|c|}
\hline Ley Ambiental & $0 \%$ & $0 \%$ & $0 \%$ & $20 \%$ & $5 \%$ \\
\hline Política Ambiental & $0 \%$ & $0 \%$ & $70 \%$ & $60 \%$ & $5 \%$ \\
\hline $\begin{array}{c}\text { Sustentabilidad y } \\
\text { Economía }\end{array}$ & $0 \%$ & $0 \%$ & $40 \%$ & $10 \%$ & $5 \%$ \\
\hline $\begin{array}{l}\text { Sustentabilidad y } \\
\text { Descentralización }\end{array}$ & $0 \%$ & $0 \%$ & $10 \%$ & $0 \%$ & $5 \%$ \\
\hline Gestión Medioambiental & $0 \%$ & $0 \%$ & $10 \%$ & $30 \%$ & $5 \%$ \\
\hline $\begin{array}{c}\text { Planeamiento y Gestión de } \\
\text { las Ciudades }\end{array}$ & $0 \%$ & $10 \%$ & $0 \%$ & $0 \%$ & $21 \%$ \\
\hline Pobreza & $0 \%$ & $0 \%$ & $10 \%$ & $10 \%$ & $5 \%$ \\
\hline Comunidades Indígenas & $0 \%$ & $10 \%$ & $0 \%$ & $10 \%$ & $\mathbf{0 \%}$ \\
\hline Movimientos Sociales & $0 \%$ & $10 \%$ & $10 \%$ & $0 \%$ & $5 \%$ \\
\hline Desarrollo Sustentable & $0 \%$ & $0 \%$ & $0 \%$ & $40 \%$ & $47 \%$ \\
\hline $\begin{array}{c}\text { Riesgo y Catástrofe } \\
\text { Urbana }\end{array}$ & $0 \%$ & $0 \%$ & $0 \%$ & $\mathbf{0 \%}$ & $16 \%$ \\
\hline Prevención y Mitigación & $0 \%$ & $0 \%$ & $0 \%$ & $\mathbf{0 \%}$ & $5 \%$ \\
\hline $\begin{array}{c}\text { Transformación y } \\
\text { Degradación Ambiental } \\
\end{array}$ & $0 \%$ & $0 \%$ & $0 \%$ & $\mathbf{0 \%}$ & $5 \%$ \\
\hline $\begin{array}{c}\text { Instrumentos Innovadores } \\
\text { de Gestión Urbana }\end{array}$ & $0 \%$ & $0 \%$ & $0 \%$ & $\mathbf{0 \%}$ & $53 \%$ \\
\hline Participación & $0 \%$ & $0 \%$ & $0 \%$ & $\mathbf{0 \%}$ & $47 \%$ \\
\hline Huella Ecológica Urbana & $0 \%$ & $0 \%$ & $0 \%$ & $\mathbf{0 \%}$ & $11 \%$ \\
\hline $\begin{array}{c}\text { Indicadores } \\
\begin{array}{c}\text { Medioambientales y de } \\
\text { Calidad }\end{array} \\
\end{array}$ & $0 \%$ & $0 \%$ & $0 \%$ & $\mathbf{0 \%}$ & $37 \%$ \\
\hline $\begin{array}{c}\text { Sistemas Complejos e } \\
\text { Integración de los } \\
\text { Instrumentos en los Planes }\end{array}$ & $0 \%$ & $0 \%$ & $0 \%$ & $\mathbf{0 \%}$ & $26 \%$ \\
\hline $\begin{array}{c}\text { Alianzas } \\
\text { Públicas/Privadas, Nuevos } \\
\text { Instrumentos Económicos } \\
\text { y Eficiencia Económica }\end{array}$ & $0 \%$ & $0 \%$ & $0 \%$ & $\mathbf{0 \%}$ & $26 \%$ \\
\hline Las Ciudades Intermedias & $0 \%$ & $0 \%$ & $0 \%$ & $\mathbf{0 \%}$ & $11 \%$ \\
\hline $\begin{array}{c}\text { La Crisis del Medio } \\
\text { Ambiente }\end{array}$ & $0 \%$ & $0 \%$ & $0 \%$ & $\mathbf{0 \%}$ & $5 \%$ \\
\hline
\end{tabular}

* Las cifras aquí representan los porcentajes de todas las referencias relevantes que ocurren en los cuatro periodos en los que aparece cada palabra clave.

\section{- Período 1970 a 1979: el medio ambiente en compartimentos}

Los temas más salientes en este período son la demografía, el agua, el aire, el suelo y la tierra, el transporte, la energía, la ecología, los residuos, el desarrollo urbano y la polución ambiental. Cada uno de estos tópicos fueron tratados como objeto de estudios separados, y la literatura de la época tampoco demuestra que exista una interrelación entre los mismos. Durante la investigación se ha identificado a este período como el del 'medio ambiente y sus compartimentos'. Desde un punto de vista histórico, este fue un 
período de rápido crecimiento en la población y un desarrollo genuino en la región. El nivel de vida alcanzó niveles altos. La cuestión de la conservación de los bosques amazónicos surgió como una de las primeras cuestiones ambientales de la época. En cuanto al ambiente urbano, encontramos referencias sobre estudios de sistemas de agua, los efectos de la producción de la energía y su uso en las ciudades, los aspectos meteorológicos de la contaminación del aire; estudios que intentan describir los efectos del rápido crecimiento y desarrollo como causa del proceso de la sustitución de importaciones, objetivo de la industrialización.

\section{- Período 1980 a 1989: implicaciones ambientales del desarrollo}

En este período los temas de mayor importancia son los impactos ambientales de los sistemas de agricultura, la ecología humana, la energía y la ecología, el transporte urbano y su polución, desarrollo comunitario y las comunidades indígenas y el medio ambiente. Varios fueron los factores que afectaron la evolución positiva de la economía de los años 70, entre ellos las grandes migraciones a las ciudades y la creciente población - como consecuencia de los procesos ocurridos en el período anterior $-\mathrm{y}$ los efectos de las dos crisis petroleras de los 70 junto con la desastrosa situación de la deuda externa que trajo grandes consecuencias para el desarrollo en toda América Latina. Los años 80 significaron una gran recesión económica. Los puestos de trabajo para los inmigrantes pobres disminuyeron considerablemente, así como el nivel de vida. Las condiciones sociales, como la raza, lengua, ingresos y las cuestiones de género, fueron determinantes en la posición social.

La crisis económica fue un factor influyente en cuanto al desarrollo de una nueva percepción del medio ambiente. Los países de América Latina se vieron confrontados con una serie de circunstancias que reflejaban el pasado, mientras que comenzaban a exhibir signos sobre las tendencias urbanas ambientales futuras. Las políticas de desarrollo del pasado comenzaron a afectar en forma negativa y a tener implicaciones para el medio ambiente durante este período. Así encontramos literatura que describe las consecuencias de las políticas de desarrollo del pasado sobre la crisis económica que afectó todos los aspectos de la vida urbana, incluyendo al medio ambiente. A modo de ejemplos, encontramos referencias que describen las implicaciones del modelo tecnológico de la agricultura; de la generación de residuos, su recolección y remoción; la calidad de las redes de suministro de agua para la creciente población urbana; las consecuencias de la explotación de recursos en áreas subdesarrolladas; la calidad de la vida urbana en la región; los impactos de las actividades humanas sobre la naturaleza, etc. 
Al mismo tiempo, una nueva tendencia en la literatura describe las consecuencias de la crisis para los movimientos sociales de América Latina. Las comunidades indígenas se hicieron escuchar después de encontrarse discriminadas en silencio durante décadas; y comenzaron a reclamar su derecho sobre la herencia natural en muchos países con el objeto de frenar la explotación en manos de la civilización. Aunque esto poco tenga que ver con el medio ambiente urbano, el fortalecimiento de los movimientos sociales hacia cuestiones ambientales ha evolucionado en una fuerza importante hacia la década del 90. Los movimientos sociales tomaron forma principalmente a escala comunitaria en las áreas más pobres de las mega-ciudades; Lima (Miranda, et al., 1996) y Río de Janeiro (Edelman, et al., 1997) son ejemplos típicos.

Finalmente, la crisis económica de América Latina en 1980 ha pasado a ser un constante recordatorio de una historia de desarrollo pobre de la planeación al ser un crónico la crisis de la deuda, aún después de que un cambio en la economía haya tenido lugar. El crecimiento económico que ha puesto bajo presión al medio ambiente urbano, también ha reconocido la importancia de la preservación de los recursos naturales. Muchos de los gobiernos de la región, por presión de algunos países desarrollados, han decidido aceptar la solución verde al problema de la deuda, por medio de lo que conocemos como 'deuda por canje de naturaleza'. Los intercambios, en este contexto, reducen la deuda nacional y preservan el ambiente. En este sentido, grupos internacionales ambientales comprarían la deuda de un país en el mercado financiero e invertir el monto al gobierno deudor para que éste proteja o crea, por ejemplo, un parque nacional, el cual estaría en manos del gobierno con la posibilidad de generar ingresos.

Desgraciadamente, ninguna de las opciones para reducir la deuda durante los años 80, ha resultado efectiva. Aunque el tema de la deuda poco tenga que ver con la cuestión del medio ambiente urbano, así y todo, hizo que la población urbana tome conciencia del valor del medio ambiente. Más aún, al final de la década la comunidad internacional tomaría conciencia sobre las interrelaciones entre los pobres y el ambiente, y la necesidad de lograr un patrón de desarrollo que por lo menos le dé a la región una chance de ser sustentable (O'Brien, 1991).

Quizá el avance más significativo en la literatura de este período para el desarrollo del campo de la gestión urbana ambiental, sea la introducción de la literatura urbana sobre el desarrollo. Mientras que en éste período toda la literatura relacionada con temas ambientales seguía enfocada sobre aspectos ecológicos, como la polución del agua, la calidad del agua, la polución del aire, la contaminación del suelo, y relacionada con regiones o países, la literatura de América Latina sobre temas de desarrollo comenzó a volcarse hacia los temas urbanos. El crecimiento tecnológico y económico, el 
incremento de la población, y las consecuencias sociales de ambos fenómenos, como la creación de empleo, era un fenómeno urbano en crecimiento, que atraía la migración urbana. Por lo tanto, la literatura urbana de esta época, se orientaba hacia temas de crecimiento y desarrollo de las ciudades, sin relacionar esta transformación histórica y social con aquella otra literatura orientada a las implicaciones ambientales de las actividades humanas. Los dos cuerpos literarios se mantuvieron como temas separados por un largo tiempo.

\section{- Período 1990 a 1992: desarrollo económico y sustentabilidad}

Con el reconocimiento de la interrelación entre la crisis económica de América Latina de los 80 y las condiciones ambientales, la literatura sobre cuestiones ambientales de la región dio un viraje. La literatura de a principios de los 90 comenzó a ser más explícita en cuanto al reconocimiento de la relación entre el desarrollo económico y sus consecuencias ambientales; mientras, los académicos especialistas y los profesionales desarrollarían nuevos modelos para explicar dichas interacciones y encontrar soluciones.

No es sorprendente encontrar una serie de libros relacionados con las diferentes percepciones de la polución y cuestiones ambientales. Las referencias bibliográficas variarían desde temas del sindicalismo y el medio ambiente, la mujer y el medio ambiente, hasta la política del medio ambiente. En esta época la literatura comienza a tomar un matiz urbano extenso de las preocupaciones ambientales que va desde cuestiones ambientales verdes, marrones y grises. Los libros relacionados con las soluciones también comenzaron a tomar una orientación hacia lo urbano. Mientras que las preocupaciones tradicionales ambientales seguían dominando la agenda, modelos computarizados sobre las interacciones entre población y medio ambiente, sobre las distintas maneras de conservar los recursos naturales y sobre las medidas para abatir la polución atmosférica, reconocerían el modelo de asentamiento urbano latinoamericano y su efecto sobre el medio ambiente.

Podemos observar en la literatura un cambio que va desde cuestiones sobre la conservación de los recursos naturales hacia cuestiones o temas que, por el contrario, intentan promover la sostenibilidad ambiental. El desarrollo sustentable se convirtió en el principio para las políticas de los gobiernos, y la descentralización pasó a ser la política alternativa y el medio para aliviar las desigualdades sociales y lograr un crecimiento sostenible.

El viraje en las tendencias literarias desde temas rurales a urbanos fue facilitado no sólo por el reconocimiento de las interrelaciones entre el desarrollo económico y las condiciones medioambientales - cada vez más urbanas- pero también por el contexto económico y político de la región. 
Poco a poco los países se fueron recuperando de las crisis económicas y dejando atrás las agrias memorias dictatoriales. Brasil y Argentina eligieron sus gobiernos otra vez, dando lugar a la democracia que guiaría los procesos de descentralización.

Dentro de este contexto, las comunidades urbanas comenzaron a pedir por el reconocimiento de sus problemas específicos, de los cuales la mayoría eran de carácter ambiental. La polución del aire y el agua proveniente de las emisiones y afluentes de las fábricas y los vehículos, así como de los residuos y excrementos, causaban serios problemas a la salud pública en áreas con menos recursos y extremadamente pobres. La falta de agua potable, tratamiento de aguas y de residuos, junto a la congestión del tráfico, el ruido y los residuos peligrosos de pequeñas industrias, se convirtieron en cuestiones de gran importancia para la población que empezó a demandar por acciones más concretas.

Durante este período surge una nueva tendencia que demuestra la relación entre el desarrollo económico y la sustentabilidad. Las consecuencias de las grandes migraciones rurales urbanas, no son sólo vistas por su importancia social y de desarrollo, sino que ahora también se reconocen los problemas ambientales como resultado de las migraciones. Este es el período en que los problemas urbanos son, por primera vez, integrados con los problemas ecológicos, y comienzan a ser vistos bajo el nombre de problemas ecológicos urbanos o urbanos ambientales. Por ejemplo, la polución del aire como consecuencia del transporte, la polución del agua como consecuencia de la mala gestión, inundaciones como consecuencia de la deforestación demandada por las urbes, problemas de salud como consecuencia de la degradación ambiental urbana, polución de ruido como consecuencia de la naturaleza y la densidad de las actividades urbanas, contaminación del suelo como consecuencia de la creciente demanda de las manufacturas basadas en áreas urbanas, etc.

\section{- Período 1993 a 1998: desarrollo sustentable}

Pareciera haber un claro corte en la literatura a partir de 1993, y luego de los efectos producidos por la Conferencia de las Naciones Unidas sobre el Medio Ambiente, llevada a cabo en Río de Janeiro en 1992. El período de transición de a principio de 1990 ya había sido superado y la estabilidad económica y política habían sido ya aceptada en la región como parte de la realidad. El concepto de desarrollo sustentable es aceptado como un objetivo global. Y la globalización y la interdependencia presionarían a los países de esta región, y a otros, para ajustarse a los estándares ambientales internacionales.

Lo que caracteriza a la literatura del presente es su enfoque hacia cuestiones de gestión ambiental. La incorporación del medio ambiente en las 
políticas públicas y la importancia del ambiente urbano ya nos son algo de prioridad. El medio ambiente es considerado ahora tener un componente rural y otro urbano y es considerado ser marrón, gris y verde. El tema de mayor importancia es cómo manejar el medio ambiente y quién debe ser la autoridad responsable. Los distintos grupos de interés ya no son sólo gobiernos nacionales y regionales, junto a sus cuerpos descentralizados, sino también grupos comunitarios que se encuentran fuera de la estructura del gobierno, formales o informales (por ej. Grupos comunitarios); invasiones organizadas a espacios urbanos vacíos realizadas por ciudadanos rurales que se re-ubican en la ciudad; el gran número de organizaciones no gubernamentales (ONGs) tan características por sus movimientos sociales en muchos países de América Latina, y por último el sector privado.

El sector privado juega un doble papel, por un lado, como contaminador que tiene que tomar medidas para contaminar menos, y por otro lado como facilitador de infraestructura urbana, en varios niveles, proveyendo servicios ambientales urbanos. Entre estos últimos, podemos mencionar, la limpieza de las calles, la recolección de los residuos y suministro de agua. En éstos, el rol del sector privado varía desde la provisión de trabajo y disminución de la pobreza al tratamiento de los residuos y remoción; en donde la eficiencia y los bajos costos para el fisco son considerados.

Finalmente, otra tendencia de importancia que surge en este período es la necesidad de proveer un marco institucional y legislativo para permitir que las iniciativas de la comunidad y de la municipalidad sean exitosas en cuanto al manejo del medio ambiente. Bolivia puede considerarse ser uno de los países que más lejos a llegado en este respecto y en esta región.

La evolución de la literatura a lo largo de los cinco periodos es clara: primero el reconocimiento de los problemas ambientales, luego la evaluación de los efectos de la polución en las grandes urbes, el reconocimiento de la sistemática interconexión entre el medio ambiente y la economía urbana de la región y consecuentemente la necesidad de un desarrollo sustentable, y por último aparecieron los intentos para crear un marco, políticas e instrumentos para el manejo del medio ambiente urbano. Sin embargo, los problemas del pasado no han sido resueltos todavía. Éstos siguen siendo sujeto de estudios y continúan formando parte de muchas publicaciones. Se entiende entonces que la variedad temática incluye temas como el desarrollo rural y el medio ambiente, la degradación ambiental de los ríos, la deforestación (temas publicados por primera vez en el Período 1); la población y el medio ambiente, incluyendo los efectos de toda clase de polución urbana sobre el ser humano (temas publicados inicialmente durante el Período 2); la íntima conexión entre la pobreza y la degradación ambiental en las ciudades, así como la necesidad de tomar medidas para asegurar un desarrollo sustentable 
(temas que hicieron su primera aparición durante el periodo 3). Aun así, los temas relacionados con el desarrollo de un marco institucional y regulatorio, y la determinación de un rol apropiado del sector privado en la gestión urbana ambiental, así como la promoción de una gestión ambiental en la industria, comienzan a formar parte de un precedente en la literatura latinoamericana (temas que evolucionaron durante el Período 4).

La frase 'desarrollo sustentable' se ha convertido en el último periodo como la frase clave. Luego del reconocimiento de la relación entre el desarrollo económico y el medio ambiente, así como la identificación de los problemas nacidos de esta relación, fue claro en la literatura que el gran tema del Siglo XXI sería el del desarrollo sustentable de las ciudades. La literatura latinoamericana de este periodo se centra en los problemas urbano ambientales que surgen como consecuencia de las actividades humanas, también se centra en las posibles soluciones, las opciones de políticas para desarrollar marcos dentro de los cuales implementar soluciones, los cambios necesarios en la gestión para apoyar la implementación, las nuevas estructuras institucionales requeridas para dar lugar a las soluciones y por último, los cambios esenciales en la conducta humana para que todo esto sea factible. En resumen, los temas ecológicos, medio ambientales y urbanos convergen como un solo tema en la literatura de esta región.

\section{- Período 1999 a 2017: de la política a la acción.}

Sin embargo, el período que abarca los últimos diecinueve años se convirtió en una era transitoria de gestión ambiental urbana, cuya conclusión fue marcada por Habitat III en Quito en 2016. Durante este período de tiempo, la literatura pasó de la consideración de conceptos y de políticas al reconocimiento que los programas y las acciones significativos para permitir el desarrollo sostenible se deben tomar en el nivel local. Además, se ha hecho evidente que estas acciones deben emprenderse de manera sistemática en el marco de la planificación urbana y regional. Por 2002, muchos países ya tenían las leyes, las instituciones e incluso algunas victorias judiciales para apoyar la gestión ambiental urbana, pero seguía habiendo serios problemas de contaminación debido a sistemas de producción y consumo que no se actualizaban a la velocidad necesaria (Rodríguez-Becerra y Espinoza, 2002). Así, el tiempo para el desarrollo de políticas ha terminado; es hora de actuar.

\section{Las tendencias en las publicaciones periódicas}

Luego de haber completado la búsqueda de la literatura española y portuguesa sobre gestión urbana ambiental y luego de haber percibido las tendencias de la misma, se llevó a cabo la segunda etapa de esta investigación. Se procedió con una búsqueda en Internet para identificar las 
revistas publicadas en la región y relacionadas con temas ambientales. El propósito de esta segunda etapa fue el de confirmar si las tendencias en las publicaciones periódicas coincidían con las tendencias de los libros. La búsqueda se realizó país por país, en Argentina, Brasil, Chile, Colombia, Cuba, México y Perú.

La primera observación arribada durante esta búsqueda es que no se encontró ninguna revista sobre temas urbano ambientales o sólo ambientales en ninguno de los países mencionados, existente antes de 1990. De hecho, la mayoría de las revistas comienzan a ser publicadas alrededor de 1992/93. Además, dada su naturaleza y materia de estudio, las revistas sobre medio ambiente en América Latina parecieran ser dirigidas a una audiencia educada, de académicos, o activistas de ONGs, así como profesionales y representantes de industrias y empresas líderes. Una audiencia interesada sobre los últimos acontecimientos en temas como casos jurídicos, legislación, eventos internacionales y estándares ambientales internacionales. Se asumió que los artículos encontrados relacionados con al ambiente urbano coinciden con los temas ambientales regionales del Período 4 (por ejemplo, temas como desarrollo sustentable, políticas económicas, descentralización, derecho ambiental y legislaciones, etc.), así como la llamada a la acción del Período 5. Este paralelismo entre la literatura y las publicaciones periódicas fue confirmado cuando se observó que todos los artículos publicados en las revistas y en los países mencionados versaban sobre los temas arriba mencionados y coincidían también con su período histórico. Una última observación es que la tendencia en las publicaciones periódicas es la misma en todos los países en que se basó esta investigación, lo cual es un punto interesante de análisis si se tiene en cuenta que dichos países varían en su nivel de desarrollo, tipo de economía, población, sistema político, estructura social, condición e ubicación geográfica.

\section{Conclusión}

Como resultado de la investigación, se puede concluir que existe una clara tendencia en la cual convergen tres elementos en la literatura sobre gestión urbana ambiental de América Latina. Estos elementos fueron evolucionando a lo largo de los cinco períodos analizados en este artículo. A tal efecto, se ha elaborado la Figura 2. Inicialmente (1970-1979), los estudios o trabajos realizados sobre problemas ecológicos $\mathrm{u}$ ambientales se diferenciaban de aquellos que analizaban temas de desarrollo y consideraciones tecnológicas. Lo mismo sucedió durante el segundo período (1980-1989), aunque comenzaban a surgir ya obras literarias más comprensivas relacionadas con el desarrollo y crecimiento de las ciudades. Durante este período temas como la economía y la población dominaban la literatura. En el tercer período (1990-1992), sin embargo, el acelerado 
crecimiento económico del período anterior y la creciente migración a las ciudades, seguido por las crisis económicas regionales y el surgimiento de los movimientos sociales comenzaban a aparecer como temas predominantes en la literatura latinoamericana. La combinación de la actividad humana y sus implicancias ambientales pasaron a formar parte del foco de atención en la mayoría de los estudios regionales del tercer período. Siguiente, los hilos en la literatura se condujeron hacia el medio ambiente, el desarrollo económico y la urbanización en América Latina, formando así el cuarto período histórico (1993-1994). Debido a las presiones externas e internas, se tomó consciencia de que las ciudades en América Latina ya no pueden controlar sus problemas ambientales si no manejan apropiadamente los procesos que los originan. Finalmente, el quinto y último período (1999 2017) se convirtió en un período de transición. Se han realizado progresos en la formulación y aplicación de políticas medioambientales, tanto a nivel nacional, subnacional y local, como en el desarrollo de diversos planes e instrumentos para la protección del medio ambiente. Además, los países tienen hoy autoridades medioambientales que presentan diferentes grados de desarrollo, y la mayoría cuentan con un organismo ejecutivo nacional especializado, ya sea en forma de Ministerio, Comité de medio ambiente u otra entidad equivalente (ibid.). Las políticas nacionales y regionales empezaron a transformarse en planes locales de acción en el marco de la planificación urbana y regional. Este enfoque evolutivo de la gestión ambiental urbana era evidente en habitat III como se indica a continuación.

Habiendo realizado todo este análisis cabe mencionar que el último paso de esta investigación es un proyecto en curso. Se espera completar de analizar la literatura de lenguas española y portuguesa escrita en América Latina y sobre América Latina poste 2017; eso es después de otro período significativo marcado por un posible habitat IV u otro acontecimiento semejantemente significativo en la planificación urbana y la gestión ambiental. Habitat III concluyó con la redacción de una nueva agenda urbana, destacada por la declaración de Quito sobre ciudades sostenibles y asentamientos humanos para todos. Se trata de un documento de referencia en la gestión ambiental urbana mediante la promesa de la sección 14. (c) ser guiado, entre otros, por el principio:

la sostenibilidad medioambiental, mediante la promoción de la energía limpia, el uso sostenible de la tierra y los recursos en el desarrollo urbano, así como la protección de los ecosistemas y la biodiversidad, incluyendo la adopción de estilos de vida saludables en armonía con la naturaleza; promover el consumo sostenible y los patrones de producción; construyendo resiliencia urbana; reducir los riesgos de desastres; y mitigar y adaptarse al cambio climático (Habitat III, 2016). 
Otra declaración de la nota es que un impulsor fundamental del cambio

es:

.... revigorizando la planificación y el diseño urbano y territorial a largo plazo e integrado con el fin de optimizar la dimensión espacial de la forma urbana y de ofrecer los resultados positivos de la urbanización.

\section{Instrucciones para futuras investigaciones}

Todas las señales apuntan, entonces, a la siguiente fase de gestión ambiental urbana siendo una de implementación a nivel local. La investigación futura determinará si éste es de hecho el caso. Las cuestiones que pueden desarrollarse durante este análisis de fase III para América Latina incluyen si y cómo se transferirán los recursos y la autoridad para la implementación de los gobiernos centrales y regionales a las localidades, si tienen la experiencia técnica y gerencial necesaria para ejecutar programas complejos y si la comunidad internacional invertirá en estos esfuerzos la terminación de un estudio similar de la literatura inglesa general , así como de estudios complementarios de la literatura que representa un número de otras idiomas y regiones, de las cuales este trabajo formaría solamente una parte, es otra dirección fructuosa para la investigación adicional. Así, en un futuro estudio las conclusiones alcanzadas para la literatura latinoamericana podrían ser comparadas a las del estudio de la literatura inglesa general, así como a ésos resultados de la literatura de otras regiones y/o de idiomas para desarrollar un análisis de la tendencia del tiempo, que indicaría la intensidad de ciertos temas (tendencias) según período y la región del tiempo. Esto no sólo indicaría el desarrollo del campo mediante la identificación de las cuestiones más importantes y posibles soluciones en cualquier momento, sino que también daría a conocer qué regiones del mundo son líderes en un aspecto particular de la gestión ambiental urbana.

\section{References:}

1. Baddeley, O., \& Fraser, E. (1989). Drawing the Line: Art and Cultural Identity in Contemporary Latin America. London: Verso.

2. Bartone, C. (1989). Environmental Issues in Urban Management. Draft INU Discussion Paper, Washington DC: The World Bank.

3. Berry, B. J. L., \& Horton, F. E. (1974). Urban Environmental Management: Planning for Pollution Control. Englewood Cliffs, NJ: Prentice-Hall.

4. Button, K. J., \& Pearce, D. W. (1989). "Improving the Urban Environment: How to Adjust National and Local Government Policy for Sustainable Urban Growth." Progress in Planning, 32, 141-161. 
5. Edelman, D. (1998). "La ciudad como ecosistema: La administración del medio ambiente y la contaminación," en R. Eduardo, \& R. Daughters (Eds.), La Ciudad en Siglo XXI (pp. 331-336). Washington DC: The Inter-American Development Bank.

6. Edelman, D. J., Procee, P., \& Acioly, C. (1997). Sustainable Urban Development and the Urban Poor in Rio de Janeiro. In the Conference: The Challenge of Environmental Management in Metropolitan Areas.

7. Fernández, R. (2000). Gestión ambiental de ciudades: Teoría crítica y aportes metológicos. México: Programa de las Naciones Unides para el Medio Ambiente.

8. Galarza, Y. and Carola, H. (1991). Saneamiento ambiental, disposicion de desechos y calidad de vida de las mujeres en Bolivia. Quito: Fundacion Natura.

9. Galilea, S. (1994). Modelo de gestión urbana para ciudades intermedias en América Latina. Santiago: CEPAL Comisión Económica para América Latina y el Caribe.

10. Giradet, H. (1992). The GAIA Atlas of Cities: New Directions for Sustainable Urban Living. London: Gaia Books Limited.

11. Goodman, D., \& Redclift, M. (1991). Environment and Development in Latin America: The Politics of Sustainability. New York, NY: Manchester University Press.

12. Gutierrez, P. J. (1991). Hay polucion en Cuba? el dano creciente al medio ambiente se ha convertido en una amenza para el futuro del hombre. Cuba: Habana.

13. Habitat III (2016). New Urban Agenda. Draft Outcome Document (10 September 2016) for Adoption in Quito, Ecuador.

14. Hardoy, J. E., \& Satterthwaite, D. (1989). Environmental Problems in Third World Cities: A Global Issue Ignored? In Conference on Cities: The Mainspring of Economic Development in Developing Countries.

15. Leitmann, J. (1994). Rapid Urban Environmental Assessment: Lessons from Cities in the Developing World. Volumes 1 and 2, Urban Management Programme Discussion Papers 14 and 15. Washington DC: The World Bank. https://doi.org/10.1596/0-82132790-9

16. Leitmann, J. (1999). Sustaining Cities: Environmental Planning and Management in Urban Design. New York, NY: McGraw-Hill.

17. Meier, R., Berman, S., Campbell, T., \& Fitzgerald, C. (1981). "The Urban Ecosystem and Resource-Conserving Urbanism," in Third World Cities (pp. 1-7, 32-44). Berkeley, CA: Institute for Urban and Regional Development. 
18. Miranda, S., Liliana, J. C., \& Palacios, J. D. (1996).” La situación de la gestión urbana ambiental en el Péru" en M. S. Liliana (Ed.), Ciudades para la Vida: Experiences exitoses y propuestas para la acción (PGU Serie Gestión Urbana No. 6). Lima: United Nations.

19. Mitlin, D., \& Satterthwaite, D. (1996). "Sustainable Development and Cities," in C. Pugh (Ed.), Sustainability, the Environment and Urbanisation. London: Earthscan.

20. O'Brien, P. J. (1991). "Debt and Sustainable Development in Latin America," in D. Goodman, \& M. Redclift (Eds.), Environment and Development in Latin America: The Politics of Sustainability. New York, NY: Manchester University Press.

21. Rees, W. (1996). "Ecological Footprints of the Future." People \& the Planet, 5, 6-9.

22. Schuster, M., \& Edelman, D. J. (2002). “Tendencias en la Gestión Urbana Ambiental en Latino América.” Contraste Regional, 2, 183204.

23. Schuster, M., \& Edelman, D. J. (2003). "Latin American Trends in Urban Environmental Management." Clean Technologies and Environmental Policy, 5, 50- 60.

24. Vásconez, S. D. (2000). "Hacia una nueva gestión ambiental urbana," en C. Fernando (Ed.), Desarrollo Cultural y Gestión en Centros Históricos (pp. 247-256). Quito: FLACSO.

25. White, R., \& Whitney, J. (1992). "Cities and Environment: An Overview," in R. Stren et al. (Eds.), Sustainable Cities: Urbanization and the Environment in International Perspective (pp. 8-59). Boulder, CO: Westview Press.

\section{Anexo: Lista de palabras clave}

La lista de palabras claves representa los temas más comunes aparecidos en la literatura latinoamericana sobre la gestión urbana ambiental entre 1970 y 1998. La ocurrencia de las palabras en la búsqueda Internet, representa los elementos claves utilizados para trazar las tendencias en la literatura. Basado sobre esto es que emergieron los cuatro periodos históricos anteriormente mencionados. Las palabras claves se describen a continuación con el objeto de clarificar el significado de la misma en la forma en que se utiliza en este artículo. La definición de cada palabra no es necesariamente la definición de la palabra en sí. Se vio necesario desarrollar la lista y sus definiciones ya que durante la búsqueda en Internet la literatura obtenida no haría referencia a la palabra clave insertada sino tal vez a temas relacionados a la misma. Figura 2, que sigue la lista de palabras clave, mencionadas a continuación son las más significantes y ocurrentes durante la búsqueda. 
- Impactos Ambientales: palabra clave que refleja los efectos sobre el medio ambiente en general y sobre varios ecosistemas como consecuencia de las actividades humanas, como ser, la agricultura, transporte, polución atmosférica, pobreza, etc.

- Demografía: en la literatura encontrada se refiere más que nada al crecimiento de la población y la expansión del espacio urbano debido a las migraciones.

- Agua: se refiere tanto a la provisión de agua y a la calidad del agua (incluyendo la polución). No se refiere al tratamiento del agua.

- Aire: generalmente referido a la polución atmosférica como consecuencia de las emisiones de $\mathrm{CO} 2$ del transporte.

- Tierra y Suelo: palabras referidas en la literatura al uso extensivo de las tierras para actividades agrícolas y sus implicaciones en la calidad del suelo.

- Transporte: se refiere tanto a los sistemas de transporte como a la polución del aire que produce.

- Energía: es una palabra clave generalmente referida a la polución de la energía como consecuencia de proyectos de infraestructura como diques, plantas eléctricas, redes de caminos, etc.

- Desarrollo Urbano: en la literatura latinoamericana usualmente se refiere a las cuestiones relacionadas con la arquitectura y el crecimiento urbano, y a la necesidad de satisfacer la creciente demanda de viviendas.

- Ecología: normalmente referido a la degradación de los ecosistemas, como ha ocurrido en el caso del Amazonas y otras áreas forestales en la región.

- Residuos: aparece en la literatura por primera vez no como residuos en sí, sino como el valor de los residuos y su importancia con respecto al medio ambiente.

- Polución Ambiental: se refiere a la polución de todos los compartimentos ambientales (ej.: aire, agua, suelo).

- Contaminación Urbana:

- Población y Medio Ambiente: término que reconoce las interrelaciones entre la población y sus alrededores.

- Salud: se refiere a la salud humana como consecuencia de la polución ambiental.

- Mujer: como tema aparece en el tercer periodo y está enfocado hacia el impacto de la polución ambiental en la vida diaria de las mujeres.

- Percepciones: se refiere a las nuevas percepciones en la vida que han ido evolucionando en relación con los problemas ambientales en general, pero más que nada, orientadas hacia el ambiente urbano (ej.: la percepción sobre la importancia del ser humano y sus alrededores y las ciudades en las que viven). 
- Ley Ambiental: se refiere a la ley ambiental nacional e internacional, la cual tiene por objeto resolver y controlar las tendencias que son dañinas para la vida humana y el medio ambiente urbano.

- Política Ambiental: dentro del contexto de este artículo el término está relacionado con los problemas ambientales asociados al crecimiento económico y a las propuestas políticas para alcanzar un desarrollo sustentable.

- Sustentabilidad y Economía: generalmente referido a los swaps (ej.: el canje entre prácticas sustentables y protección del medio ambiente por una reducción de la deuda externa).

- Sustentabilidad y Descentralización: tema característico del tercer periodo histórico mencionado en este artículo. Generalmente se refiere a la descentralización geográfica de la actividad económica, considerada como una solución para aliviar las desigualdades sociales. En el presente, sin embargo, el término estaría más relacionado con la descentralización política.

- Gestión Medioambiental: usado en la literatura para referirse a la gestión ambiental en el sentido técnico y también para describir los enfoques institucionales y de gobierno, así como sus métodos para manejar los problemas ambientales.

- Planeamiento y Gestión de las Ciudades: surge en el segundo periodo de este artículo y en relación con los aspectos físicos, ecológicos y paisajísticos de las áreas urbanas.

- Pobreza: descripta en la literatura sólo como parte de la compleja cadena entre conservación, problemas ambientales y desarrollo socioeconómico.

- Comunidades Indígenas: término relacionado con los problemas ambientales y las prácticas sustentables ejercitadas por las comunidades indígenas. Generalmente esto se relaciona con el caso del Amazonas y los impactos de la civilización moderna sobre la vida de los indígenas.

- Movimientos Sociales: palabra clave que hace referencia a los movimientos sociales iniciados como consecuencia del reconocimiento de la importancia del medio ambiente. El término está relacionado con los impactos de las actividades humanas sobre sectores discriminados de América Latina (ej. comunidades indígenas, y los pobres).

- Desarrollo Sustentable: por un lado, se define claramente en relación con crecimiento económico y la problemática ambiental; por otro lado, el término es utilizado para contrastar el actual estilo de desarrollo con las necesidades de una región analizando las conexiones múltiples entre la economía y la ecología para luego filosofar sobre el futuro utópico de América Latina. 
- Riesgo y Catástrofe Urbana: en América Latina, la gestión del riesgo y el desastre urbano ha sido un movimiento reaccionario que ha impulsado diferentes niveles de gobierno para evaluar y abordar el riesgo. Las secuelas de los desastres ambientales, la vulnerabilidad de los nuevos asentamientos, el tamaño de las ciudades y las presiones internacionales han impulsado a los municipios y a los gobiernos nacionales a hacerse cargo de estas cuestiones.

- Prevención y Mitigación: son actividades que tienen como objetivo estabilizar la concentración de gases de efecto invernadero en la atmósfera. Los principales esfuerzos en América Latina han girado en torno a abordar cuestiones de deforestación, mecanismos de desarrollo sostenible "desarrollo limpio", eficiencia energética, gestión de residuos urbanos, créditos verdes, impuestos sobre el carbono, política de energía renovable, campañas alternativas de energía y combustibles orientadas al usuario, mejoras en el transporte público, planificación de la tierra, mejoras tecnológicas en la agricultura, programas de agua residencial y programas dirigidos a la alta industria contaminante.

- Transformación y Degradación Ambiental: la discusión sobre la gestión ambiental urbana se refiere comúnmente a la degradación como el daño ambiental causado por la rápida conversión de la tierra natural en un área urbanizada para la formación de asentamientos.

- Instrumentos Innovadores de Gestión Urbana: la literatura latinoamericana sobre gestión ambiental urbana ha evolucionado a partir de la discusión de políticas y su marco a más estudios incluyendo la tecnología como herramienta para ayudar a alcanzar los objetivos de sostenibilidad/medio ambiente. Esto abarca estudios que utilizan SIG (sistemas de información geográfica), planificación tecnócrata, toma de decisiones guiadas por datos, innovación a través de nuevas herramientas de financiamiento, sofisticados índices, uso de certificados o incentivos de reforestación "certificados de incentivo forestal", evaluación de costos ecológicos y indicadores cualitativos de progreso como el examen de la calidad de vida.

- Participación: en la bibliografía se pone de manifiesto un mayor énfasis en la inclusión de la participación pública y la consulta pública en la toma de decisiones a nivel local/regional, así como en el ámbito nacional. La participación incluye también decisiones públicas, liderazgo consistente, inclusión de voces poblacionales vulnerables, así como autoridades locales y consulta pública.

- Huella Ecológica Urbana: se refiere al capital natural necesario para sustentar el crecimiento urbano en las ciudades latinoamericanas.

- Indicadores Medioambientales y de Calidad: son parámetros y medidas que aportan cuantitativamente una visión de los temas 
cualitativos comunes como el estado del medio ambiente con respecto a los aspectos de su naturaleza ecológica, sostenibilidad centrada en el ser humano, etc.

- Sistemas Complejos e Integración de los Instrumentos en los Planes: refleja un cambio progresivo al uso de los planes a nivel local y nacional para ayudar a integrar en un sistema holístico, forma integrada, sistémica diferentes aspectos de la gestión ambiental para permitir un discurso complejo e interdependiente la gestión de los temas medioambientales en las ciudades latinoamericanas.

- Alianzas Públicas/Privadas, Nuevos Instrumentos Económicos y Eficiencia Económica: consultar soluciones creativas a la inestabilidad financiera en el plano local como resultado de la importante presencia del sector privado en el crecimiento económico de las ciudades de América Latina, que permite la formulación de políticas para la gestión ambiental urbana.

- Las Ciudades Intermedias: es un término comúnmente utilizado para abordar medianas ciudades, que a veces son el producto de rápido crecimiento causada por la migración de las zonas rurales a las urbanas.

- La Crisis del Medio Ambiente: es desencadenada por una infraestructura obsoleta, fenómenos tales como El Niño o La Niña, la vulnerabilidad de la propia construcción y asentamiento en la explotación inadecuada de la tierra; estos son costosos y eventos fatales que se han movido los municipios y los gobiernos nacionales a pensar más seriamente sobre la gestión ambiental de las ciudades de América Latina. 